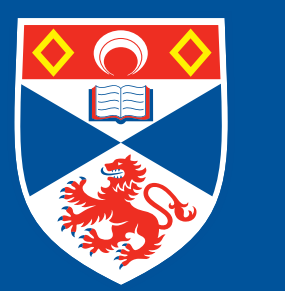

University of

St Andrews

A Risk Map of Markups: Why We D Observe Mixed Behaviors of $+$ $r$ Markups

(D)

in Seong-Hoon Kim and Seongman Moon

O

$\mapsto$

$\forall$

$\varangle$

5

D

官

$\stackrel{0}{\circ}$

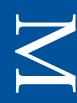

ט

ค

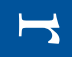

0

(D)

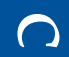

O

官

0

官

$\mapsto \cdot$

ค

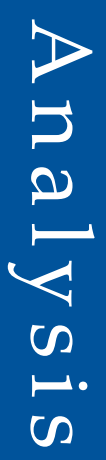

CDMA Working Paper Series No. 1409

1 Dec 2013

JEL Classification: D21, E32

Keywords: markups, risk internalization, technology, market power, cost channel, hedging channel 


\title{
A Risk Map of Markups: Why We Observe Mixed Behaviors of Markups*
}

\author{
Seong-Hoon $\mathrm{Kim}^{\dagger} \quad$ Seongman Moon ${ }^{\ddagger}$
}

December 2013

\begin{abstract}
This paper proposes an explanation for mixed evidence on the behaviors of markups. The key mechanism consists of two complementary channels of risk internalization that arise when firms face uninsurable business risks. One channel is based on passive risk consideration, through which firms raise prices to abide by riskier business thereby associating higher production with higher prices. The other channel is based on active risk management, through which firms lower prices to handle riskier business thereby associating higher production with lower prices. The relative responsiveness of the two channels to a shock depends on each firm's fundamental characteristics and leads to a sharp division of markup cyclicality across sectors.
\end{abstract}

Key words: markups, risk internalization, technology, market power, cost channel, hedging channel

JEL Classification: D21, E32

\footnotetext{
*Financial supports from the Scottish Institute for Research in Economics (SIRE) and from the Spanish Plan Nacional de I+D+I (ECO 2010-19357) are gratefully acknowledged.

†economicsnote@gmail.com; Centre for Dynamic Macroeconomic Analysis, University of St Andrews, Fife, United Kingdom, KY16 9AR.

${ }^{\ddagger}$ smoon@eco.uc3m.es; Department of Economics, Universidad Carlos III de Madrid, Calle Madrid 126, 28903 Getafe Madrid, Spain.
} 
How markups move, in response to what, and why, is almost terra incognita for macro. (...) $[\mathrm{W}] \mathrm{e}$ are a long way from having either a clear picture or convincing theories, and this is clearly an area where research is urgently needed. — Blanchard (2009, p.220)

\section{Introduction}

Economists have studied the behavior of markups first because it illuminates the characteristics of market structure and second because it clues to the nature of business cycles. For example, theories based on procyclical competition at the industry level predict markups to fall during booms and thus leave room for monetary expansion to have less pressure on inflation. Theories that predict markups to rise during booms would have different implications on the nature of business cycles and policy consequences. Data, however, show mixed evidence on the behavior of markups. For example, markups in Textiles (SIC code 22) and in Apparel (SIC code 23) behave differently over the US business cycle (Rotemberg and Woodford (1991, Table 8.b; p.109)). Basu and Fernald (1997), Gopinath et al. (2011) and De Loecker and Warzynski (2012) have also documented sectoral and locational differences in markups. The aim of this paper is to add an explanation for how and why the markups may behave differently across sectors thereby leading to unclear and mixed cyclicality as observed in data.

Our theory of markups takes the presence of uninsurable business risks to the front of story. Asset markets are incomplete over goods market outcomes arising stochastically from business operation: General Motors safeguards its business from losses due to various natural disasters but cannot prevent losses incurred through normal business outcomes. The Real Greek, a small restaurant in London, holds a similar portfolio of insurance policies. The restaurant loss-proofs its business against unprecedented flood but is exposed to unprecedented customer visits. The managers will then internalize their uninsurable business risks by usual means of pricing and production. We model uninsurable business risks within a simple price-setting newsvendor environment and derive two complementary channels of risk internalization that the managers would use: passive risk 
consideration versus active risk management. ${ }^{1}$

Passive risk consideration means to abide by business risks and leads firms to raise prices when production becomes riskier. In a world where firms set prices and produce outputs before the realization of their market demand, they will take account of the full distribution of contingencies that follow their pricing and production decisions. Each of the contingent outcomes bears either of two risk consequences: excess supply versus excess demand. Firms internalize these risks into their pricing and production and add the shadow cost of the risks to the traditional marginal cost of production. One important implication is that, even in competitive markets, the additional cost forms wedges between prices and the traditional marginal costs. Consequently, the price-quantity relation showing a firm's willingness to supply comprises the additional cost schedule ridden over the traditional supply curve. In this paper, to avoid confusion, we refer to this extended supply curve as 'offer curve'. It slopes upward in the conventional price-quantity coordinate, reflecting the principle that the higher risk a firm must bear, the higher price it wants to charge. Let us call this generalized principle of marginal cost pricing 'effective cost channel', or, in short, 'cost channel'. Indeed, this passive channel of risk internalization has been known in the various contexts: for example, Prescott (1975), Carlton (1979), Eden (1990), Rotemberg and Summers (1990), and Greenwald and Stiglitz (1993).

Active risk management means to handle business risks and leads firms to lower prices when production becomes riskier. According to the cost channel stated above, firms would associate higher output levels with higher prices to the extent that higher output implies an increase in the risk of excess supply. However, higher output levels lower the risk of excess demand at the same time. If the firm attaches economic values to lost sales opportunities arising in excess demand states, it will lower prices up to the economic value of the risk of excess demand. In general, firms will seek for a profit-maximizing balance of risks between exclusive events. Along the optimal way of balancing these risks, we derive another price-quantity relationship wherein higher output levels are associated

\footnotetext{
${ }^{1}$ As named after the metaphorical example of a street-corner newsstand, a typical price-setting newsvendor model assumes that firms choose prices and order stocks before the realization of market demand. See Petruzzi and Dada (1999) for a review of the newsvendor literature.
} 
with lower prices to offset one risk against the other. Let us refer it to a 'hedge curve', and call the generalized principle of risk-balancing pricing 'hedging channel'. This hedging channel would naturally arise where firms access to incomplete spanning of assets over their stochastic goods market outcomes. However, it has been largely neglected in the literature, if not totally. Previous works that have some touch on the risk management role of pricing include Greenwald and Stiglitz (1989), Aguirregabiria (1999), and Petruzzi and Dada (1999).

The two risk internalization channels work together and determine optimal responses of pricing and production to changing economic conditions. Suppose a common shock that raise all firms' valuation on their unsold products and thus makes them more tolerant towards possible occurrence of excess supply states. Through the cost channel, this reduction in the risk of excess supply makes firms willing to produce more for every given price (i.e., the offer curve shifts downward). At the same time, however, the initial balance between the excess supply and excess demand risks breaks down due to the shock. Consequently, through the hedging channel, firms will rebalance them at a new optimum by taking more excess supply risk relative to excess demand risk and thereby charge higher prices for every given level of output (i.e., the hedge curve shifts upward). One can see the two channels react together uniformly pushing up output but counteracting each other in price dimension. As a result, the level of output rises across all sectors whereas the precise direction of price movements can differ sector by sector.

We take the two risk internalization channels to the analysis of optimal markup behaviors. A key condition determining whether markups rise or fall during booms concerns the relative responsiveness of the two channels to changing economic conditions. In turn, the responsiveness of the channels relies on the way in which firm's fundamentals such as technology and market power are translated into the riskiness of business environment. Intuitively, firms producing perishables and facing elastic market demand tend to be more worried about unsold products and thus take on a relatively smaller excess supply risk than those firms producing nonperishables and facing inelastic market demand, other things being equal. The former firms will raise prices in response to the aforementioned 
shock since the shock makes them less anxious about excess supply states for given demand distribution. On the contrary, the latter firms tend to be more concerned about lost sales opportunities in excess demand states and thus will lower prices. As a result, with constant marginal costs normalized across sectors, one will see markups procyclical for firms producing perishables and operating in more competitive environment and countercyclical for firms producing nonperishables and operating in more concentrated markets.

Our theory of markups translates technology and market power into riskiness of business environment and provides a consistent explanation for the mixed evidence of countercyclical and procyclical behaviors at the aggregate and disaggregate levels. Since markups behave differently across sectors, the aggregate behavior conditional to economywide shocks will depend on the entire distribution of sectors. To the extent that countries are different overall in their cost and market structures, markups may also behave differently at the country level. Moreover, the aggregate markup behavior would have somewhat different properties over a long horizon as an economy experiences measurable shifts in its competition structure, for example, due to institutional and legislative changes.

\section{Literature Review}

In the literature, the two channels of risk internalization have not been brought into a unified framework. Regarding the passive channel of risk internalization, this paper shares the idea of effective marginal cost pricing with Eden (1990), Rotemberg and Summers (1990), Greenwald and Stiglitz (1993), and Dana (1998, 1999). In this literature, firms facing uncertain demand schedules add up the shadow cost of risk to the traditional marginal cost of production. For example, Eden (1990) shows that if we expand the commodity space to include sales probabilities, the optimality condition generalizes the standard marginal cost pricing.

Regarding the active risk management role of pricing in goods market, we find three 
relevant works. In the newsvendor literature of operations research, Petruzzi and Dada (1999) attempt to reconcile different specifications of demand uncertainty (additive or multiplicative to price) by invoking the role of pricing in choosing coefficient of variation of random demand schedules and show the presence of price premium above the certainty benchmark price regardless of demand specifications. Their work and the present paper share the device of modelling business risk. Aguirregabiria (1999) also considers the price-setting newsvendor problem with fixed ordering costs, which naturally induce the classical $(s, S)$ inventory rule. In this model, as a theory of clearance sales, the fixed ordering costs increase the stockout risk and make unwanted accumulation of inventories unavoidable and thereby, when combined with additional price adjustment costs, help to explain the coexistence of large periods without nominal price changes and short periods with massive price markdowns. Our work nests such an intrinsic relation between pricesetting and stockout risk. Greenwald and Stiglitz (1989) take a more direct approach to the active risk management in goods markets. To explain real price rigidity, their work employs the notion of "portfolio" within the mean-variance analysis and derives a firm's optimal decision comprising two decision instruments, pricing and production, which differ in their expected returns and risks. Our work shares the same spirit and idea with theirs, but differs much in the way of dealing with business risks in goods market. Above all, while the present paper shares either modelling device or ideas of managing risk, it identifies two distinct complementary channels of risk internalization within a single framework.

Our objective is to explain the behaviors of markups using the two channels of risk internalization. The proposed mechanism is in sharp contrast to the existing literature. Much of the existing explanation resorts solely to the waxing and waning of market power. Within this view, the rate of markup tends to increase in the size of seller's market power. For example, studies based on a game theoretic approach attribute the cyclical markups to the cyclical variations of collusion among competitors (e.g., Green and Porter (1984), Rotemberg and Saloner (1986), and Rotemberg and Woodford (1991, 1992)). Studies based on switching costs, habit formation, etc., attribute the cyclical markups to 
the cyclical variations of the elasticity of demand (e.g., Bils (1989), Klemperer (1995), Bagwell (2004), and Ravn, Schmitt-Grohe, and Uribe (2006)). Some studies suggest that markups move up and down due to the economies of scale in shopping technologies or in information dissemination that relatively weaken seller's market power when shopping intensity is high (e.g., Warner and Barsky (1995) and MacDonald (2000)). All these works share common in their reliance on market power subject to cyclical or seasonal variations. In our theory of markups, market power remains limited and indirect in its role for the determination of markup cyclicalities and works only by its implications on the size of risk. ${ }^{2}$

\section{The Business Environment}

Consider a simple price-setting newsvendor environment of Petruzzi and Dada (1999) where a risk-neutral monopolistic firm sets price $p$ and produces output $y$ before the realization of market demand $d$. Market demand is subject to exogenous random demand factor $x$ as follows:

$$
d=x D(p)
$$

where price $p>0$ and $x$ is drawn from the probability distribution function $F(x)$ with a support $[\underline{x}, \bar{x}], 0<\underline{x}<\bar{x}<\infty$, and a finite expected value. $F(\cdot)$ is continuous and differentiable with $f(x)=\frac{\partial F(x)}{\partial x}>0$ on the interior of the support. $D(\cdot)$ is bounded below by zero and twice-continuously differentiable with $D_{p}<0$. Let $\epsilon(p)=-\frac{p D_{p}(p)}{D(p)}$, the price elasticity of demand. It is assumed that $\epsilon_{p}(p)=\frac{\partial \epsilon(p)}{\partial p} \geq 0$. This condition implies the standard property that marginal revenue is strictly decreasing. ${ }^{3}$ Nevertheless, it

\footnotetext{
${ }^{2}$ Still noteworthy are some rare works that illuminate alternative mechanisms of markup pricing other than by cyclical market power. Chevalier and Scharfstein (1996) look into a link between capital market imperfection and goods market pricing. In their work based on customer markets, firms having difficulty in raising external funds during recessions raise markups as a means of helping cash flow while sacrificing their current customer stock. Markup pricing can be also seen as an instrument of marketing in loss-leader models, whereby multiproduct firms (or retailers) strategically select high demand items and sacrifice their profit margins at discounted prices to boost customers visits: see, for example, Lal and Matutes (1994) and Chevalier, Kashyap, and Rossi (2003).

${ }^{3}$ In a certainty model of monopolist with known $x$, having marginal revenue strictly decreasing in the level of output requires that $\frac{D(p) D_{p p}(p)}{\left[D_{p}(p)\right]^{2}} \leq 2$. Since $\epsilon_{p}(p)=p\left[\frac{D_{p}(p)}{D(p)}\right]^{2}\left\{\frac{1}{\epsilon(p)}-\frac{D(p) D_{p p}(p)}{\left[D_{p}(p)\right]^{2}}+1\right\}$, the condition $\epsilon_{p}(p) \geq 0$ holds if and only if $\frac{D(p) D_{p p}(p)}{\left[D_{p}(p)\right]^{2}} \leq \frac{1}{\epsilon(p)}+1$. Consequently, for $\epsilon(p)>1$ around the
} 
is noteworthy that in our model, the condition serves to limit the exercise of a firm's monopolistic market power (for it states that an increase in prices induces more elastic market demand) and thus underlies the risk-hedging role of pricing and production.

We assume the standard specification of technology: Production is subject to constant returns to scale. Let $c$ denote a constant marginal cost of production and $y$ the level of output. However, since pricing and production are made before market demand realizes, firms will find themselves either in the state of excess demand (underproduction) or excess supply (overproduction). Let $n=(y-x D(p))^{+}$denote the level of unsold products being defined by the difference between production and realized demand, where $n=y-x D(p)$ for $y>x D(p)$ and $n=0$ otherwise. Let $h$ denote a constant value of unsold products per unit. In a static environment, the economic value of unsold products could take on fixed clearance price net other related costs. In a dynamic environment, it could be due to the expected present value of inventories, which depends on various factors such as the nature of products (e.g., automobiles vs. salads), the level of storage technology, the innovation speed of production technology, financing methods and aggregate economic conditions that affect shareholder's required rate of returns, and so on. In either environment, the expected value of unsold products forms a kind of reservation value at which firm is indifferent between selling or retaining products. For expositional convenience, this paper presents a static model. Indeed, our static model is a reduced-form version which, given our purpose, has an equivalent to a multiperiod model in recursive dynamic environment under certain conditions. Our derivation of two risk internalization channels also holds with general specification of production and inventory technologies having twice-continuously differentiability and second-order regularity.

Profits are given by the total sales revenue minus the total cost, which can be expressible as a function of price $p$, output $y$, and the realized value of $x$ :

$$
\pi(p, y, x)=p\{y-n\}-c y+h n
$$

neighborhood of monopolistic pricing and production, the condition implies the standard property that marginal revenue is strictly decreasing. 
where $n=(y-x D(p))^{+}$. Assume the monopolist maximizes its expected profit: $\int_{\underline{x}}^{\bar{x}} \pi(p, y, x) d F(x)$.

Let $\hat{x}$ define the lowest admissible value of the realized $x$ needed to clear the market: $\hat{x}=\min \left[\arg _{x}\{n=0\}\right]$. Since $d$ in $(1)$ is increasing in $x$, this definition is equivalent to having a value of $x$ such that

$$
y=\hat{x} D(p)
$$

for given pair of $(p, y)$. Using the threshold value $\hat{x}$, the expected profit is expressible as

$$
\int_{\underline{x}}^{\bar{x}} \pi(p, y, x) d F(x)=\int_{\underline{x}}^{\hat{x}} p x D(p) d F(x)+\int_{\hat{x}}^{\bar{x}} p y d F(x)-c y+\int_{\underline{x}}^{\hat{x}} h n d F(x),
$$

because the sales volume $(y-n)$ contingent to the realization of $x$ is given by $y-n=x D(p)$ for $x \in[\underline{x}, \hat{x})$ and $y-n=y$ for $x \in[\hat{x}, \bar{x}]$.

\section{Risk Channels of Pricing and Production}

This section establishes two risk channels of pricing and production. Assuming the existence of an interior solution to (3), we will focus on a connection between the sufficient condition for interior maxima and the economic principles behind the risk channels of pricing and production.

\subsection{The Optimality Conditions}

With identity $(2),(p, y)$ and $(p, \hat{x})$ are one-to-one interchangeable (injective mapping) and in particular $y$ is monotone-increasing in $\hat{x}$ for given $p .{ }^{4}$ Henceforth, we conduct our analysis over the coordinate $\{(p, \hat{x})\}$. Under the existence assumption of an interior solution, we substitute (2) into (3), reformulate the expected profit as a function of $p$ and

\footnotetext{
${ }^{4}$ It is important to understand that the threshold $\hat{x}$ will be implied by the monopolist's pricing and production decisions. However, ex ante, it works like a "point estimator" imparted by the Bayesian decision theory (for example, the minimum expected loss estimator) and therefore is incorporated into a decision problem.
} 
$\hat{x}$, and name the function $W$ :

$W(p, \hat{x})=\int_{\underline{x}}^{\hat{x}} p x D(p) d F(x)+\int_{\hat{x}}^{\bar{x}} p \hat{x} D(p) d F(x)-c \hat{x} D(p)+\int_{\underline{x}}^{\hat{x}} h\{\hat{x} D(p)-x D(p)\} d F(x)$.

The finite integrals above are well-defined and differentiable in $(p, \hat{x})$ since $D$ is continuous and differentiable in $p$ and the space for random demand factor $x$ is nonempty, compact-valued, and continuous $(0<\underline{x}<\bar{x}<\infty)$. Applying the Leibniz rule to this reformulated maximization problem, we obtain the first order conditions as follows:

$$
\left\{\begin{aligned}
W_{\hat{x}}(p, \hat{x}) & =\int_{\hat{x}}^{\bar{x}} p D(p) d F(x)-c D(p)+h \int_{\underline{x}}^{\hat{x}} D(p) d F(x) & =0 \\
W_{p}(p, \hat{x}) & =\int_{\underline{x}}^{\hat{x}}\left[x D(p)+p x D_{p}(p)\right] d F(x)+\int_{\hat{x}}^{\bar{x}} \hat{x}\left\{D(p)+p D_{p}(p)\right\} d F(x) & \\
& +h \int_{\underline{x}}^{\hat{x}}\{\hat{x}-x\} D_{p}(p) d F(x)-c \hat{x} D_{p}(p) & =0 .
\end{aligned}\right.
$$

A sufficient condition for a local maximum to exist is as follows:

Lemma 1. A pair of price and estimate that satisfies $W_{\hat{x}}(p, \hat{x})=0$ and $W_{p}(p, \hat{x})=0$ constitutes a local maximum of $W(p, \hat{x})$ under the condition that $\eta(\hat{x}) \epsilon(p)>\frac{p}{p-h}$ where $\eta(\hat{x})=-\frac{\partial\{1-F(\hat{x})\}}{\partial \hat{x}} \frac{\hat{x}}{1-F(\hat{x})}=\frac{\hat{x} f(\hat{x})}{1-F(\hat{x})}$.

Proof. See Appendix. Essentially, we show the link between the two stated conditions and the second order sufficient condition for the existence a local maximum. We will provide an economic interpretation for these conditions after Proposition 2.

Notice first that the condition $W_{\hat{x}}(p, \hat{x})=0$ in $(5)$ can be reduced to

$$
\underbrace{c}_{\text {MC.Y }}=\underbrace{p\{1-F(\hat{x})\}}_{\text {MR.Y.XD }}+\underbrace{h F(\hat{x})}_{\text {MR.Y.XS }}
$$

since $D>0$. Also observe that the condition $W_{p}(p, \hat{x})=0$ can be reduced to

$$
\underbrace{\hat{x} D(p)\{1-F(\hat{x})\}}_{\text {MR.P.XD }}+\underbrace{\int_{x}^{\hat{x}}\left[x\left\{D(p)+(p-h) D_{p}(p)\right\}\right] d F(x)}_{\text {MR.P.XS }}=0,
$$


since $W_{p}(p, \hat{x})=\int_{\hat{x}}^{\bar{x}} \hat{x} D(p) d F(x)+\int_{\underline{x}}^{\hat{x}}\left[x\left\{D(p)+(p-h) D_{p}(p)\right\}\right] d F(x)+\frac{D_{p}(p)}{D(p)} W_{\hat{x}}(p, \hat{x})$. Here, we attach a set of acronyms to help interpret the mathematical terms in the language of economics:

- MC.Y: marginal cost of production,

- MR.Y.XD: expected marginal revenue w.r.t. production decision over excess demand states,

- MR.Y.XS: expected marginal revenue w.r.t. production decision over excess supply states,

- MR.P.XD: expected marginal revenue w.r.t. pricing decision over excess demand states,

- MR.P.XS: expected marginal revenue w.r.t. pricing decision over excess supply states.

MC stands for marginal cost; MR for marginal revenue; Y for w.r.t. production; $\mathrm{P}$ for w.r.t. pricing; XD for excess demand; and XS for excess supply. Additionally, we will shorthand the risk associated with XD states by XD-risk, and the risk associated with XS states by XS-risk, because the business risks in the present model primarily take the form of XD versus XS. XS-risk is basically captured by the probability of XS states,

$\int_{\underline{x}}^{\hat{x}} d F(x)=F(\hat{x})$, and XD-risk by the probability of XD states, $\int_{\hat{x}}^{\bar{x}} d F(x)=1-F(\hat{x})$. Given the distribution function $F(\cdot)$, choosing $\hat{x}$ determines the relative size of XS-risk and XD-risk.

\subsection{Cost Channel}

As directly read from the acronyms, the risk characterization of the two optimality conditions can, in principle, be made in the language of marginal analysis. Equation (6) is the standard equalization condition between the marginal revenue and the marginal cost of production. It states that, for a chosen price, the level of ex ante optimal production will be found where the marginal cost equals the sum of the expected marginal revenues. 
Again, each of marginal revenue terms is associated with one of the two distinct demand states, XD and XS. Conditional on XD states, one additional unit of production leads to an additional revenue exactly by the chosen price. Ex ante, the economic value of the additional revenue is captured by the term MR.Y.XD. Conditional on XS states, one additional unit of production means one additional accumulation of unsold products, whose economic value is captured by the term MR.Y.XS.

Let us rewrite (6) as follows:

$$
p=(1+\underbrace{\frac{F(\hat{x})}{1-F(\hat{x})}}_{\text {price-cost wedge }})\{c-h F(\hat{x})\} .
$$

The price-quantity relation in this new expression is the one that has been proposed by Prescott (1975), Eden (1990), Rotemberg and Summers (1990), and Dana (1998, 1999), who applied the idea of effective marginal cost pricing to the producer decision problem under demand uncertainty. As previously named, $c$ is the traditional marginal cost directly related to production decision (MC.Y), and $h F(\hat{x})$ is the expected marginal revenue w.r.t. production decision over excess supply states (MR.Y.XS), which can be seen as an expected reservation value. Obviously, their difference must be positive at an interior optimum: $c>h F(\hat{x})$. At the same time, it holds that $p>c$ at an interior optimum because otherwise the firm would not have produced. So we will see $p>$ $h F(\hat{x})$ at optimum, which is related to a non-speculation condition to rule out speculative motivation such as producing for hoarding not for sales (i.e., to rule out intentional sales refusal).

As a whole, expression (8) demonstrates that the firm prices up over the direct production cost by the odds of XS states, $\frac{F(\hat{x})}{1-F(\hat{x})}$. As the firm increases production, the firm has to bear higher XS-risk. As XS-risk increases, the odds also go up. As the odds go up, the firm charges a higher price. Put this mechanism directly in a Bayesian decision context, a firm (a player) doing business (play) by taking on larger odds would internalize the risk by increasing the price (prize) that it receives if it sells (succeeds). In short, the marginal cost-revenue equalization condition (8) shows a firm's willingness to supply 
in the presence of uninsurable business risk, whereby the cost of the risks overrides the traditional supply curve. We name condition (8) as an offer curve so that one can have the same intuition but avoid confusion with the traditional supply curve.

Proposition 1 (Offer curve). Given a non-degenerate distribution $F(\cdot)$, the set of pairs $(p, \hat{x})$ that satisfies condition (6) generates an upward-sloping curve over the plane $\{(p, \hat{x})\}$.

Proof. See Appendix.

The passive risk consideration is seen in this proposition, which essentially states that the higher the risk a firm faces, the higher the price it wants to mark up. For given economic environment, this principle deduces a positive slope of the offer curve. It also underlies the mechanism that leads the offer curve to react to a changing business environment. For example, through this channel, a monopolist will lower prices for every given production level when there is a favorable shock to production technology or to reservation value for each given $\hat{x}$ : If such a shock hits, the offer curve shifts downward.

\subsection{Hedging Channel}

Equation (7) states that, for a chosen level of production, the firm should set a price at which the two expected marginal revenues offset. Conditional on XD states (i.e., conditional on that it sells as many as it produces), a firm could raise the revenue by $y=$ $\hat{x} D(p)$ if it chose price higher. Thus the expected marginal revenue equals the quantity times the probability of XD, as shown in the term MR.P.XD. However, in XS states, it could lose the revenue, whose amount depends on how sensitively demand responds at each possible XS state. Thus the expected value of additional losses across XS states equals the term MR.P.XS as stated. Consequently, the firm's optimal pricing decision presented by equation (7), enunciated in MR.P.XD + MR.P.XS $=0$, seeks to balance the two contrasting risks; XD-risk and XS-risk. In other words, the firm neutralizes the business risks by hedging one risk against the other. Let us name equation (7) as a hedge curve. 
Proposition 2 (Hedge curve). Given a non-degenerate distribution $F(\cdot)$, the set of pairs $(p, \hat{x})$ that satisfies condition ( $(7)$ generates a downward-sloping curve over the plane $\{(p, \hat{x})\}$ under the condition that $\eta(\hat{x}) \epsilon(p)>\frac{p}{p-h}$.

Proof. See Appendix.

The given condition has been established as the second order sufficient conditions by Lemma 1. Indeed, it generalizes the standard textbook statement that a monopolist never chooses price in the inelastic range of market demand $(\epsilon(p)>1)$. Notice first that the term $\eta(\hat{x})=-\frac{\partial\{1-F(\hat{x})\}}{\partial \hat{x}} \frac{\hat{x}}{1-F(\hat{x})}$ is the elasticity of the probability of XD w.r.t. $\hat{x}$ : that is, how much the probability of XD decreases in response to an increase in $\hat{x}$. Now observe that $h$ is the reservation value at the margin. ${ }^{5}$ So $p-h$ is a measure of expected marginal loss in revenues conditional on XS states at optimum. And the current price $p$ amounts to marginal loss in revenues conditional on XD states at optimum. Consequently, we can think of the ratio $\frac{p}{p-h}$ as a measure of the relative marginal loss between XD and XS states found at optimum: An infinitesimal change in the optimal estimate $\hat{x}$ at the border of XS and XD states would result in an infinitesimal change in production for each given price $p$. Since such a small increase (decrease) in production leads an XD (XS) state to switch to an XS (XD) at the optimum, the firm's optimal production decision will reflect their relative impacts on its expected profits. The relative marginal loss is greater than or equal to one: $\frac{p}{p-h} \geq 1$, where the equality holds if unsold products have no value.

All in all, the given condition states that the firm's optimal pricing-production decision will not be found in the inelastic range of the XD probability and market demand. Intuitively, the monopolist will keep producing as long as additional output has little impact the XD probability and will keep raising price as long as additional price margin has little impact on market demand. We summarize this condition as follows:

\footnotetext{
${ }^{5}$ It can be shown that the given condition is expressible as $\eta(\hat{x}) \epsilon(p)>\frac{p}{p-H_{n}(0)}$ when taking a general specification for the total economic value of unsold products, where $H_{n}(\cdot)$ is the first order derivative of a twice-continuously differentiable function $H(n)$ for $n \geq 0$ with $H_{n}>0, H_{n n} \leq 0$, and $H(0)=0$. For such a general case, $H_{n}(0)$ can be read as the reservation value at the border of XD and XS states. However, for the virtue of simplicity, we take a constant reservation value throughout the paper and thus $H_{n}(n)=h$ for all $n \geq 0$.
} 


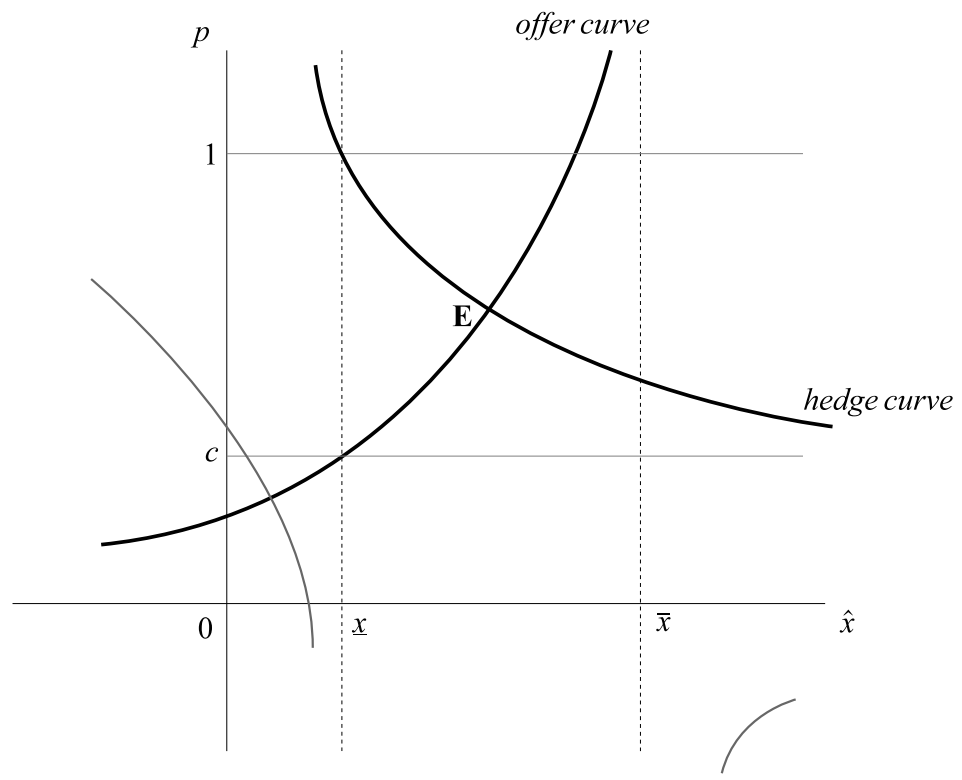

Figure 1: Example 1

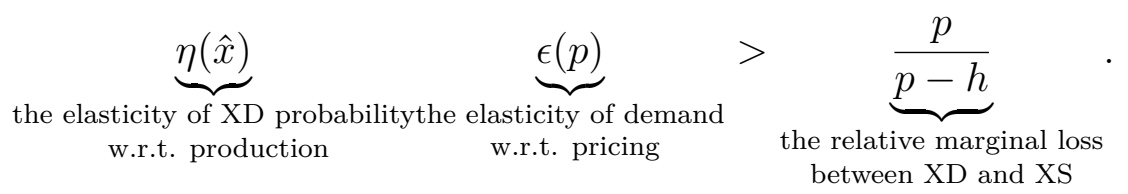

This is a generalization of the well-known elasticity condition in a certainty model of monopolist.

For given business environment, the principle of the active risk management leads to a negative slope of the hedge curve, along which the two contrasting risks offset each other. The same principle underlying Proposition 2 also applies when the business environment changes and directs the reaction of the hedge curve to such a shock. For example, when there is a shock that reduces the XS-risk for every given production level by affecting the reservation value of products, a monopolist who wants to rebalance XS- and XD-risks will take on relatively more XS-risk by increasing output for every given price (or raising prices for every given production level). In brief, through this channel, the hedge curve shifts outward (or upward) in response to such a shock.

We finalize this section with an example that illustrates how the hedge and offer curves jointly determine an optimal pair of pricing and production for given business environment.

Example 1. Let us consider a monopolist that faces market demand $d=x(1-p)$, 
with $p \in[0,1]$ and $x$ uniformly distributed over $[\underline{x}, \bar{x}]$. Goods are produced at a constant marginal cost $c \in(0,1)$ and completely perishable yielding $h=0$. Under the assumptions, the offer curve is given by $p=c\left\{\frac{\bar{x}-\underline{x}}{\bar{x}-\hat{x}}\right\}$ following from (6), and the hedge curve $p=$ $1-\frac{\hat{x}^{2}-\underline{x}^{2}}{2\left(\bar{x} \hat{x}-\underline{x}^{2}\right)}$ from (7). As shown in Figure 1, the offer curve is upward-sloping and the hedge curve is downward-sloping. Solving the two simultaneous equations, one can obtain an optimal solution $(p, \hat{x})$ where the two curves meet. As indicated by $\mathbf{E}$ in Figure 1 it is unique over the joint space $[0,1] \times[\underline{x}, \bar{x}]$.

\section{A Risk Map of Technology and Market Power}

By inversely relating pricing to production, the hedge curve seeks to neutralize the two forms of XS and XD risks each other. By positively relating pricing to production, the offer curve seeks to weigh the effective cost of the risks. The same principles of the active risk management (hedging channel) and the passive risk consideration (cost channel) can be applied to the ways in which the hedge and offer curves react to changing business environment. Through the two risk internalization channels, the fundamentals like technology and market power can be translated in terms of riskiness of business environment. This section studies the behavior of markups and examines how the fundamental differences in technology and market power affect the level and cyclicality of markups.

To this aim, we normalize the fundamental difference across sectors and configure a continuum of sectors as follows: With respect to technology, we use the ratio $a=h / c \in A$ to characterize relative "technology" differentiable across sectors. Sectors located near $a=0$ would be those who produce hardly storable goods. With respect to market power, we consider demand with a constant elasticity $\epsilon$ and use its inverse $m=1 / \epsilon \in M$ (the Lerner index) to characterize relative "market power" differentiable across sectors. As $m \rightarrow 0$, markets approach to perfect competition. Sectors close to $m=1$ are those who have extreme monopoly power. Now mapping sectors to their relative technology and market power $\{(a, m)\}$, the entire world of business in our model is represented by a rectangular space $A \times M$. 


\subsection{Markups: Risk and Power}

Let us map a continuum of sectors onto the space of $A \times M$ in which every sector $(a, m)$ is currently at its optimum. Given its fundamentals $(a, m)$, each sector's optimal (gross) markup rate $\mu=p / c$ and optimal estimate $\hat{x}$ satisfying (6) and (7) are related as follows:

$$
\mu=\frac{1}{1-\underbrace{m}_{\text {market power }} \underbrace{R(\hat{x})}_{\text {risk factor }}}
$$

where

$$
R(\hat{x})=\frac{\hat{x}}{T(\hat{x})}\{1-F(\hat{x})\}+F(\hat{x})
$$

with

$$
T(\hat{x})=\int_{\underline{x}}^{\hat{x}} x d F(x \mid x<\hat{x})=\frac{\int_{\underline{x}}^{\hat{x}} x d F(x)}{F(\hat{x})} .
$$

Equation (10) decomposes optimal markups into two parts: (i) the traditional measure of market power, $m=1 / \epsilon$, and (ii) another component attributable to riskiness of business environment, $R(\hat{x})$. Let us call $R(\hat{x})$ "risk factor". Notice that for a standard model of monopolist with known $x=\hat{x}$, the risk factor equals one, $R(\hat{x})=1$ since $F(\hat{x})=1$. So

the optimal (gross) markup rate $\mu=\frac{1}{1-m}$ : For the certainty case, the markup rate is solely a direct reflection of market power. For stochastic demand cases, the risk factor deviates above from 1: $R(\hat{x})>1$.

The risk factor lies at the center of a firm's hedging motive between XD and XS risks. Basically, it is the weighted average of $\frac{\hat{x}}{T(\hat{x})}$ and 1 with a weight vector of the XD and XS probabilities. In turn, $\frac{\hat{x}}{T(\hat{x})}$ can be thought as a "conditional sales ratio" between XD and XS states. Notice that it can be deductively written as follows:

$$
\frac{\hat{x}}{T(\hat{x})}=\frac{\frac{\int_{\hat{x}}^{\bar{x}} \hat{x} d F(x)}{1-F(\hat{x})}}{\frac{\int_{\underline{x}}^{\hat{x}} x d F(x)}{F(\hat{x})}}=\frac{\int_{\hat{x}}^{\bar{x}} p \hat{x} D(p) d F(x \mid x \geq \hat{x})}{\int_{\underline{x}}^{\hat{x}} p x D(p) d F(x \mid x<\hat{x})} .
$$

Apparently, the numerator of the last expression is the expected sales revenue conditional on XD states and the denominator is the expected sales revenue conditional on XS states. A brief digression to finance will help to appreciate the economic meanings behind this 
conditional sales ratio. Consider an index that shows the relative size of the expected values of one's investment portfolio between mutually exclusive sets of events (e.g., booms and recessions). An investor would optimally select it by choosing the composition of assets and the total investment size. Similarly, we can think of the conditional sales ratio here as an index of relative revenue between the two mutually exclusive sets of events (XD versus XS). A firm will choose it by combining two decision instruments (pricing and production) where the two risk channels agree and thus the offer curve meets the hedge curve. Moreover, the firm will choose different conditional sales ratios over time as its business environment changes, like an investor would do as the economic phases move on.

Having the conditional sales ratio in it, the risk factor equation (11) shows an optimal way of balancing of the XD and XS risks. Having the risk factor in it, the optimal markup rule (10) implies that the risk factor and the market power jointly determine the behavior of markups in response to changing business environment.

\subsection{Sectoral Difference in the Markup Behavior}

Firm's cost structure is determined by both its idiosyncratic technological constraints and aggregate economic conditions (for example, cyclical movements in oil prices and wage rates, etc.). It is also natural to attribute one's market power to a combination of its business characteristic and the economywide competition factors (for example, changes in import tariffs and fair-trade clauses, etc.). In this section we will draw our attention to the case of aggregate technology shock that affects every sector and conduct a comparative statics analysis to study its impact and implication on the behavior of markups across different sectors. However, one can easily conduct a similar analysis for the case of a shock to the economy's overall competition structure.

Let $a^{\prime}$ denote a firm's new technological position following a common real shock $q$. Without loss of generality, we assume that the shock is multiplicative to its fundamentals: $a^{\prime}=q a$. Initially we set $q=1$ and then allow small variations in the neighborhood. Suppose a shock to $q$ that leads to $q>1$ and thus raises each firm's technological 
position from $a$ to $a^{\prime}$. Since $a=h / c$ as defined, one can think of such a shock as a common improvement in production or in storage conditions (i.e., reduction in production cost $c$ or rise in the reservation value $h$ ). Such a favorable common shock $q$ raises each individual firm's expected marginal revenues associated with both pricing and production in XS states (MR.P.XS and MR.Y.XS) and reduces the XS-risk of holding one additional unit of unsold product. Therefore, through the cost channel, firms become willing to produce more for every given $p$. In brief, the offer curve shifts downward. One can easily confirm it by equation (6). At the same time, since the initial balance between the XS and XD risks breaks down due to such a shock, firms will have rebalanced them at a new optimum by taking more XS-risk relative to XD-risk. Consequently, through the hedging channel, firms become willing to charge higher price for every given $\hat{x}$. In brief, the hedge curve shifts upward. One can easily confirm it by equation (7). Having the reactions of the two channels together, we find them uniformly pushing up $\hat{x}$ but counteracting each other in $p$ dimension. By implication, the level of output rises across all sectors whereas the precise movement of markups needs a further investigation sector by sector.

Lemma 2. For any distribution function $F(x)$, which is non-degenerate, continuous on the closed interval $[\underline{x}, \bar{x}]$ with $0<\underline{x}<\bar{x}<\infty$, and differentiable on the open interval $(\underline{x}, \bar{x})$, the risk factor $R(\hat{x})$ is non-monotone in $\hat{x}$ and positively (negatively) sloped in the neighborhood of the lower bound $\underline{x}$ (the upper bound $\bar{x}$ ).

Proof. See Appendix. Essentially, we show that $-\infty<\lim _{\hat{x} \uparrow \bar{x}} R_{\hat{x}}(\hat{x})<0<\lim _{\hat{x} \downarrow \underline{x}} R_{\hat{x}}(\hat{x})<$ $\infty$, where $R_{\hat{x}}(\hat{x})=\frac{d R}{d \hat{x}}$; and then call for the mean value theorem to claim the existence of at least one point $\hat{x}_{0} \in(\underline{x}, \bar{x})$ such that $R_{\hat{x}}\left(\hat{x}_{0}\right)=0$.

This result is highly general and accommodates cases for which some arbitrary distribution function might make the risk factor have multiple peaks. However, the risk factor has a single peak for most of the continuous distribution functions used in practice (e.g., Gamma, Log-normal, Weibull, Uniform, etc.). Henceforth, we draw our attention to the single-peaked risk factor case with the family of regular distribution functions. Then the continuum of sectors can be divided into two groups: whether each member sector's 
optimal $\hat{x}$ lies below or above a certain value at which the risk factor has a unique peak. Of course, it depends on each sector's initial location $(a, m)$ of the fundamentals. Intuitively, firms who produce easily perishable products and face highly elastic demand (i.e., relatively small $a$ and small $m$ ) tend to choose relatively small $\hat{x}$. Firms with relatively large $a$ and large $m$ tend to choose relatively large $\hat{x}$.

This sectoral division upon the fundamentals is better appreciated by recalling the sufficient condition established in Lemma 1, which requires to hold that $\eta(\hat{x}) \epsilon(p)>\frac{p}{p-h}$ at an interior optimum. As previously discussed, this condition generalizes the well-known elasticity condition of a standard monopolist model and states that optimal pricingproduction decision will not be found in the inelastic range of the XD probability and market demand. It can be now rewritten in terms of the sectoral location $(a, m)$ as follows:

$$
\eta(\hat{x})>m \frac{\mu}{\mu-q a} .
$$

Obviously, as we travel from sectors with relatively small $a$ and $m$ to those with relatively large $a$ and $m$ (i.e., towards north-east direction from somewhere in south-west on $A \times$ $M)$, we will see them choose larger and larger $\eta(\hat{x})$ (i.e., more and more elastic XD probability), which in turn implies larger and larger $\hat{x}$ at their own optimum. Recall that this condition is employed by Proposition 2 to establish the hedging channel of pricing and production. It links now the sectoral fundamentals $(a, m)$ directly to the principle of active risk management via the non-monotone property of the risk factor. We will see below that the non-monotonicity of the risk factor has an important implication for the behaviors of markups across sectors.

Proposition 3 (Risk map). Consider a world of monopolistic markets $A \times M$ as described above, where every sector $(a, m)$ is currently at its optimum. The world $A \times M$ is partitioned according to the property of markups for each sector $(a, m)$ in response to a common shock, $q$, that disturbs all the sectors in the neighborhood of their initial optima.

Proof. First, we already know from Lemma 2 that for any continuous distribution function $F(x)$, there is at least one point $\hat{x}_{0} \in(\underline{x}, \bar{x})$ such that $R_{\hat{x}}\left(\hat{x}_{0}\right)=0$, and $R_{\hat{x}}(\hat{x})>0\left(R_{\hat{x}}(\hat{x})<\right.$ 
0 ) around the neighborhood of the lower bound $\underline{x}$ (the upper bound $\bar{x}$ ). Second, for those sectors $\{(a, m)\}$ whose initial optimal $\hat{x}$ are found where $R$ is positively (negatively) sloped, a common shock leading to a higher optimal $\hat{x}$ will result in a higher (lower) value of $R$ at a new optimum. Third, we also know from (10) that a higher level of $R$ implies a higher markup rate for every given level of $m$. Consequently, for the common shock $q$, some sectors reduce markups while some raise.

Central to this result is the optimal adjustment of the risk factor which is nonmonotone across sectors in response to changing economic conditions (summarized by changes in $q$ in the present model). As previously discussed, an increase in $q$ raises optimal $\hat{x}$ for every sector. The rise of optimal $\hat{x}$ has two effects on the risk factor. First, it means to rebalance the XS- and XD- probabilities by increasing the XS-probability with a commensurable decrease in the XD-probability. For a small rise in optimal $\hat{x}$, the change in the risk factor due to rebalancing probabilities is given by $f(\hat{x})\left\{1-\frac{\hat{x}}{T(\hat{x})}\right\}$. Notice that this direct effect reduces the risk factor since the conditional sales ratio $\frac{\hat{x}}{T(\hat{x})}$ is greater than 1. Second, on the other hand, the conditional sales ratio itself is subject to change in response to changing economic conditions. This second effect raises the risk factor since the conditional sales ratio is increasing in $\hat{x}$.

The two effects on the risk factor sum to

$$
R_{\hat{x}}(\hat{x})=\underbrace{f(\hat{x})\left\{1-\frac{\hat{x}}{T(\hat{x})}\right\}}_{(-)}+\underbrace{\left[\frac{\hat{x}}{T(\hat{x})}\right]^{\prime}\{1-F(\hat{x})\}}_{(+)},
$$

whose sign will differ across sectors at their optimal path. Lemma 2 implies that the second effect is large enough to offset the first effect for the sectors $\{(a, m)\}$ who have chosen relatively small initial values for their optimal $\hat{x}$. As a result, these sectors will raise their risk factor at optimum in response to a favorable common shock, and therefore exhibit a procyclical behavior of markups as implied by (10). As we travel towards those sectors whose optimal $\hat{x}$ 's are relatively large, we will see their markups countercyclical in response to an increase in $q$ because the second effect diminishes and the slope of the risk factor turns negative. 


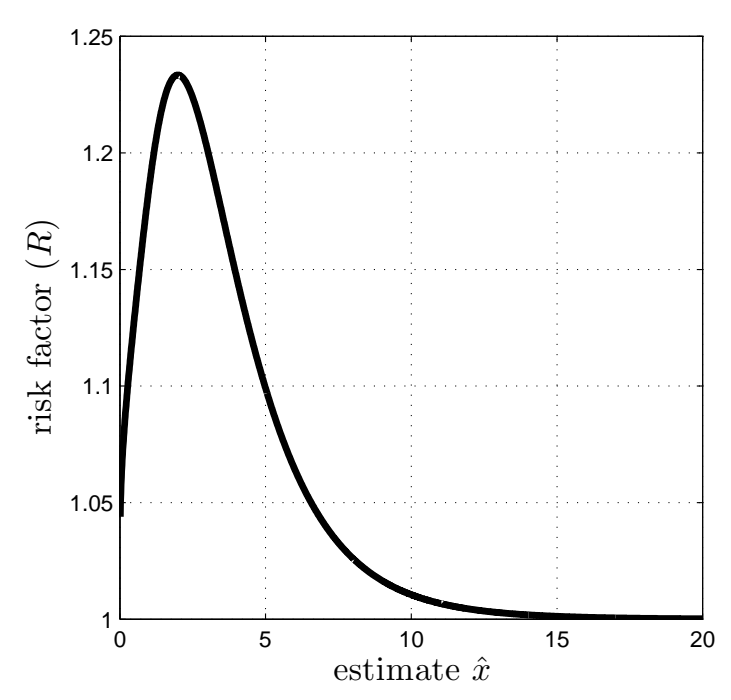

(a) Risk factor

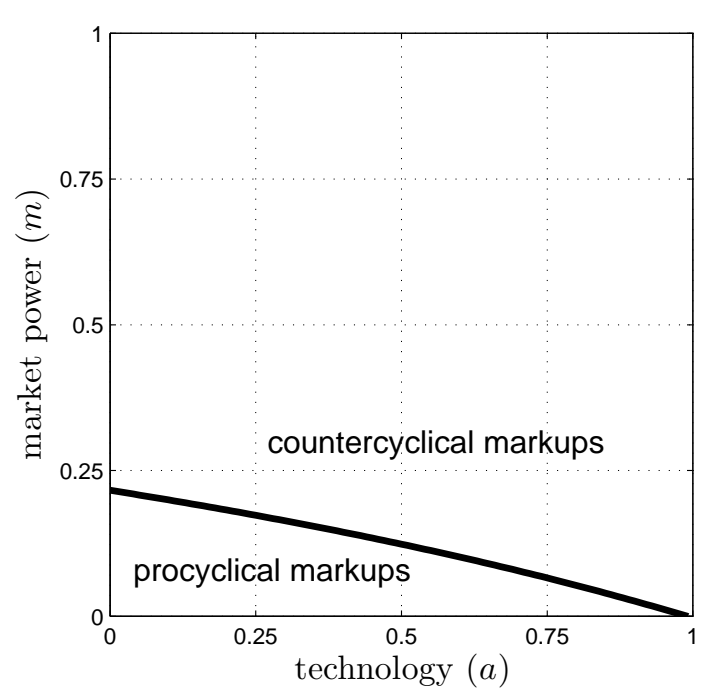

(b) Risk map of markups

Note: The figures are based on $q=1.01$ and a log-Normal distribution for $x$ with mean 1 and standard deviation 0.5 for all the sectors $(a, m) \in A \times M=(0,1)^{2}$.

Figure 2: Example 2

The next example serves as an illustration of Proposition 3.

Example 2. Assume $x$ follows a log-Normal distribution. Figure 2 is based on a logNormal with mean 1 and standard deviation 0.5 and the common technology $q=1.01$ (i.e., $1 \%$ increase from 1 to 1.01). Figure 2 a draws the risk factor $R(\hat{x})$ with respect to optimal $\hat{x}$ for each given sector $(a, m)$. Figure $2 \mathrm{~b}$ partitions the space of technology $a$ and market power $m$ into two areas: one area associates a set of fundamentals $\{(a, m)\}$ with the cases in which firms optimally respond to a small increase in $q$ by lowering their prices, and the other area associates another set of fundamentals with the cases in which it is optimal to raise prices. The separation curve collects those sectors $\{(a, m)\}$ whose initial optimum implies the maximal level of $R(\hat{x})$ or $R_{\hat{x}}(\hat{x})=0$.

In this example, we map a continuum of sectors onto a unit rectangle $A \times M=(0,1)^{2}$, by which it is assumed that the reservation value of products cannot exceed production cost $(0<a<1)$ and the elasticity of demand is greater than one and less than infinity $(0<m<1)$. Two caveats are as follows: First, by comparing the relative size of the two areas above and below the curve in Figure 2b, one might be tempted to read our analysis suggestive of dominance of countercyclical markups. However, in the literature, 
the most popular range of calibration on the elasticity parameter $\epsilon$ is between 4 and 10 (for example, Chari, Kehoe, and McGrattan (2000) set it to 10 and Gali (2008) uses 6): that is, the usual calibration range of market power $m$ runs from 0.1 to 0.25 . Therefore, to technically speak, the two areas are almost the same in size. Second, the precise location of the separation curve can vary with the initial value of $q$ and the distribution function $F$, although the results essentially remain intact.

\section{Conclusions}

Firms have access to insurance policies that help them safeguard their business from losses due to various natural disasters but cannot trade a complete set of claims that are contingent on losses incurred through their usual business operation. This paper identifies two complementary channels of risk internalization that firms would use. One channel is based on the passive risk consideration, through which the managers raise prices to abide by riskier business and thereby associates higher production with higher prices. The other channel is based on the idea of active risk management, through which the managers lower prices to handle riskier business and thereby associates higher production with lower prices.

We take the two risk internalization channels to the analysis of markups and show that the behavior of markups differs across sectors depending on the way in which each firm's fundamentals like technology and market power are translated into riskiness of business environment. Where the two channels are at work, a shock to business environment results in an asymmetry in optimal price adjustment across sectors: It leads to countercyclical adjustment of price-cost markups in sectors producing nonperishable goods with high market power, and procyclical adjustment in others having the opposite characteristics of the fundamentals.

This mixed behavior at the disaggregate level suggests that the aggregate markups will depend on the precise distribution of firms on grids of technology and market power. Two implications are immediate: First, if a country comprises more non-farm business 
and more concentrated industries than others, the country will be more likely to show countercyclical markups at the aggregate level relative to other countries. Second, if an economy experiences pro-competition institutional changes, the economy will be more likely to show procyclical markups at the aggregate level than before. Although this prediction about cross-sectional and over-time behavioral difference is based on a highly stylized model, the ultimate implication is clear: One will continue to observe mixed behaviors of markups. 


\section{References}

Aguirregabiria, Victor. 1999. "The Dynamics of Markups and Inventories in Retailing Firms." The Review of Economic Studies 66 (2):275-308.

Bagwell, Kyle. 2004. "Countercyclical Pricing in Customer Markets." Economica $71(284): 519-542$.

Basu, Susanto and John G. Fernald. 1997. "Returns to Scale in U.S. Production: Estimates and Implications." Journal of Political Economy 105 (2):249-283.

Bils, Mark. 1989. "Pricing in a Customer Market." The Quarterly Journal of Economics $104(4): 699-718$.

Blanchard, Olivier. 2009. "The State of Macro." Annual Review of Economics (1):209228.

Carlton, Dennis W. 1979. "Contracts, Price Rigidity, and Market Equilibrium.” Journal of Political Economy 87 (5):1034-1062.

Chari, V. V., Patrick J. Kehoe, and Ellen R. McGrattan. 2000. "Sticky Price Models of the Business Cycle: Can the Contract Multiplier Solve the Persistence Problem?" Econometrica 68 (5):1151-1179.

Chevalier, Judith A., Anil K. Kashyap, and Peter E. Rossi. 2003. "Why Don’t Prices Rise during Periods of Peak Demand? Evidence from Scanner Data." American Economic Review 93 (1):15-37.

Chevalier, Judith A. and David S. Scharfstein. 1996. "Capital-Market Imperfections and Countercyclical Markups: Theory and Evidence." American Economic Review $86(4): 703-725$.

Dana, James D. 1998. "Advance-Purchase Discounts and Price Discrimination in Competitive Markets." Journal of Political Economy 106 (2):395-422. 
1999. "Equilibrium Price Dispersion under Demand Uncertainty: The Roles of Costly Capacity and Market Structure." The RAND Journal of Economics 30 (4):632660.

De Loecker, Jan and Frederic Warzynski. 2012. "Markups and Firm-Level Export Status." American Economic Review .

Eden, Benjamin. 1990. "Marginal Cost Pricing When Spot Markets Are Complete." Journal of Political Economy 98 (6):1293-1306.

Gali, Jordi. 2008. Monetary Policy, Inflation, and the Business Cycle: An Introduction to the New Keynesian Framework. Princeton University Press.

Gopinath, Gita, Pierre-Olivier Gourinchas, Chang-Tai Hsieh, and Nicholas Li. 2011. "International Prices, Costs, and Markup Differences." American Economic Review $101(6): 2450-2486$.

Green, Edward J. and Robert H. Porter. 1984. "Noncooperative Collusion under Imperfect Price Information." Econometrica 52 (1):87-100.

Greenwald, Bruce C. and Joseph E. Stiglitz. 1989. "Toward a Theory of Rigidities." American Economic Review 79 (2):364-369.

—. 1993. "Financial Market Imperfections and Business Cycles." The Quarterly Journal of Economics 108 (1):77-114.

Klemperer, Paul. 1995. "Competition When Consumers Have Switching Costs: An Overview with Applications to Industrial Organization, Macroeconomics, and International Trade." Review of Economic Studies 62:515-539.

Lal, Rajiv and Carmen Matutes. 1994. "Retail Pricing and Advertising Strategies." The Journal of Business 67 (3):345-370.

MacDonald, James M. 2000. "Demand, Information, and Competition: Why Do Food Prices Fall at Seasonal Demand Peaks?" Journal of Industrial Economics 48 (1):27-45. 
Petruzzi, Nicholas C. and Maqbool Dada. 1999. "Pricing and the Newsvendor Problem: A Review with Extensions." Operations Research 47 (2):183-194.

Prescott, Edward C. 1975. "Efficiency of the Natural Rate." Journal of Political Economy $83(6): 1229-1236$.

Ravn, Morten, Stephanie Schmitt-Grohe, and Martin Uribe. 2006. "Deep Habits.” Review of Economic Studies 73:195-218.

Rotemberg, Julio and Garth Saloner. 1986. "A Supergame-Theoretic Model of Price Wars during Booms." The American Economic Review 76 (3):390-407.

Rotemberg, Julio and Michael Woodford. 1991. "Markups and the Business Cycle." Macroecomics Annual 6:63-129.

- 1992. "Oligopolistic Pricing and the Effects of Aggregate Demand on Economic Activity." The Journal of Political Economy 100 (6):1153-1207.

Rotemberg, Julio J. and Lawrence H. Summers. 1990. "Inflexible Prices and Procyclical Productivity." The Quarterly Journal of Economics 105 (4):851-874.

Warner, Elizabeth J. and Robert B. Barsky. 1995. "The Timing and Magnitude of Retail Store Markdowns: Evidence from Weekends and Holidays." The Quarterly Journal of Economics 110 (2):321-352. 


\section{Appendix}

\section{Proof of Lemma 1}

We will show that under the stated conditions, the second order total differential $d^{2} W$ at $(p, \hat{x})$ satisfying the first order conditions is negative definite: $W_{p p}<0, W_{\hat{x} \hat{x}}<0$, and $W_{p p} W_{\hat{x} \hat{x}}-W_{p \hat{x}} W_{p \hat{x}}>0$.

Let

$$
\left\{\begin{array}{l}
L^{h}(p, \hat{x})=\hat{x} D\{1-F(\hat{x})\}+\int_{\underline{x}}^{\hat{x}} x\left[D+(p-h) D_{p}\right] d F(x) \\
L^{o}(p, \hat{x})=p\{1-F(\hat{x})\}-c+\int_{\underline{x}}^{\hat{x}} h d F(x),
\end{array}\right.
$$

where $D=D(p)$ and $D_{p}=D_{p}(p)$. In Appendix, we will continue to use these reduced notations to save space. We can then rewrite the first order conditions as follows:

$$
\left\{\begin{array}{l}
W_{p}=L^{h}(p, \hat{x})+\hat{x} D_{p} L^{o}(p, \hat{x})=0 \\
W_{\hat{x}}=D L^{o}(p, \hat{x})=0
\end{array}\right.
$$

And we obtain the second order partial derivatives:

$$
\left\{\begin{array}{l}
W_{p p}=L_{p}^{h}(p, \hat{x})+\hat{x} D_{p p} L^{o}(p, \hat{x})+\hat{x} D_{p} L_{p}^{o}(p, \hat{x}) \\
W_{p \hat{x}}=L_{\hat{x}}^{h}(p, \hat{x})+D_{p} L^{o}(p, \hat{x})+\hat{x} D_{p} L_{\hat{x}}^{o}(p, \hat{x}) \\
W_{\hat{x} p}=D_{p} L^{o}(p, \hat{x})+D L_{p}^{o}(p, \hat{x}) \\
W_{\hat{x} \hat{x}}=D L_{\hat{x}}^{o}(p, \hat{x})
\end{array}\right.
$$

where

$$
\left\{\begin{array}{l}
L_{p}^{h}(p, \hat{x})=\hat{x} D_{p}\{1-F(\hat{x})\}+\int_{\underline{x}}^{\hat{x}} x\left[2 D_{p}+(p-h) D_{p p}\right] d F(x) \\
L_{\hat{x}}^{h}(p, \hat{x})=D\{1-F(\hat{x})\}+\hat{x}(p-h) D_{p} f(\hat{x}) \\
L_{p}^{o}(p, \hat{x})=1-F(\hat{x}) \\
L_{\hat{x}}^{o}(p, \hat{x})=-p f(\hat{x})+h f(\hat{x}) .
\end{array}\right.
$$

It can be easily confirmed that $W_{p \hat{x}}=W_{\hat{x} p}$. Apparently, the signs of $\left\{L_{p}^{h}, L_{\hat{x}}^{h}, L_{p}^{o}, L_{\hat{x}}^{o}\right\}$ hold a key to the property of $d^{2} W$. 
First, make conjecture $\left\{L_{p}^{h}<0, L_{\hat{x}}^{h}<0, L_{p}^{o}>0, L_{\hat{x}}^{o}<0\right\}$. Observe also that $\left\{L^{h}(p, \hat{x})=0\right.$ and $\left.L^{o}(p, \hat{x})=0\right\}$ if and only if $\left\{W_{p}(p, \hat{x})=0\right.$ and $\left.W_{\hat{x}}(p, \hat{x})=0\right\}$ since $D>0$. Accordingly, it is straightforward to confirm $\left\{W_{p p}<0, W_{\hat{x} \hat{x}}<0, W_{p p} W_{\hat{x} \hat{x}}-\right.$ $\left.W_{p \hat{x}} W_{p \hat{x}}>0\right\}$ under the conjecture.

Next, we complete our proof by verifying the conjecture. It is immediate that $L_{p}^{o}>0$ and $L_{\hat{x}}^{o}<0$. What remains is to show that $L_{\hat{x}}^{h}<0$ and $L_{p}^{h}<0$.

(i) $\quad L_{\hat{x}}^{h}<0$ : Observe

$$
\begin{aligned}
L_{\hat{x}}^{h}(p, \hat{x}) & =D\{1-F(\hat{x})\}+\hat{x}(p-h) D_{p} f(\hat{x}) \\
& =D\{1-F(\hat{x})\}\left[1+\left\{\frac{(p-h) \hat{x} D_{p}}{\hat{x} D} \frac{\hat{x} f(\hat{x})}{1-F(\hat{x})}\right\}\right] \\
& =D\{1-F(\hat{x})\}\left[1-\frac{(p-h)}{p} \eta(\hat{x}) \epsilon(p)\right] .
\end{aligned}
$$

Since $D\{1-F(\hat{x})\}>0$, the stated condition is equivalent to that $L_{\hat{x}}^{h}(p, \hat{x})<0$ on the interior.

(ii) $L_{p}^{h}<0$ : Let us rewrite $L_{p}^{h}(p, \hat{x})$ using the fact $L^{h}(p, \hat{x})=0$ :

$$
L_{p}^{h}(p, \hat{x})=-\int_{\underline{x}}^{\hat{x}}\left[\frac{\hat{x} D_{p}}{\hat{x} D} x\left\{D+(p-h) D_{p}\right\}\right] d F(x)+\int_{\underline{x}}^{\hat{x}}\left[x\left\{2 D_{p}+(p-h) D_{p p}\right\}\right] d F(x) .
$$

Using the definition of the price elasticity of demand, we have

$$
L_{p}^{h}(p, \hat{x})=\int_{\underline{x}}^{\hat{x}} D_{p} x\left\{\frac{(p-h)}{p} \epsilon(p)+1+\frac{(p-h)}{p} \frac{p D_{p p}}{D_{p}}\right\} d F(x) .
$$

Rearranging it,

$$
L_{p}^{h}(p, \hat{x})=\int_{\underline{x}}^{\hat{x}} D_{p} x \frac{(p-h)}{p}\left\{\epsilon(p)+1+\frac{p D_{p p}}{D_{p}}\right\} d F(x)+\int_{\underline{x}}^{\hat{x}} D_{p} x \frac{h}{p} d F(x) .
$$

The first term is weakly negative because $D_{p}<0$ and $\epsilon(p)+1+\frac{p D_{p p}}{D_{p}} \geq 0$ as implied by the assumption $\epsilon_{p}(p) \geq 0$. The second term is strictly negative because $D_{p}<0$. 


\section{Proof of Proposition 1}

Let $\left.\frac{d p}{d \hat{x}}\right|_{\text {offer curve (6) }}$ denote the total derivative of $p$ w.r.t. $\hat{x}$ while holding equation (6). It is immediate that

$$
\left.\frac{d p}{d \hat{x}}\right|_{\text {offer curve (6) }}=-\frac{L_{\hat{x}}^{o}(p, \hat{x})}{L_{p}^{o}(p, \hat{x})}>0,
$$

from the proof of Lemma 1. Therefore, the offer curve slopes upward.

\section{Proof of Proposition 2}

Let $\left.\frac{d p}{d \hat{x}}\right|_{\text {hedge curve (7) }}$ denote the total derivative of $p$ w.r.t. $\hat{x}$ while holding equation (7). It is immediate that

$$
\left.\frac{d p}{d \hat{x}}\right|_{\text {hedge curve (7) }}=-\frac{L_{\hat{x}}^{h}(p, \hat{x})}{L_{p}^{h}(p, \hat{x})}<0
$$

from the proof of Lemma 1. Therefore, the hedge curve slopes downward.

\section{Proof of Lemma 2}

Let $R_{\hat{x}}(\hat{x})=\frac{d R}{d \hat{x}}$. From (11), we have

$$
R_{\hat{x}}(\hat{x})=f(\hat{x})-\frac{\hat{x}}{T(\hat{x})} f(\hat{x})+\left(1-\frac{T_{\hat{x}}(\hat{x})}{T(\hat{x})} \hat{x}\right)\left\{\frac{1-F(\hat{x})}{T(\hat{x})}\right\}
$$

where $T_{\hat{x}}(\hat{x})=\frac{\partial T(\hat{x})}{\partial \hat{x}}=\frac{\{\hat{x}-T(\hat{x})\} f(\hat{x})}{F(\hat{x})}$. Essentially, we will show that $-\infty<\lim _{\hat{x} \uparrow \bar{x}} R_{\hat{x}}(\hat{x})<$ $0<\lim _{\hat{x} \downarrow \underline{x}} R_{\hat{x}}(\hat{x})<\infty$; and then call for the mean value theorem to claim the existence of at least one point $\hat{x}_{0} \in(\underline{x}, \bar{x})$ such that $R_{\hat{x}}\left(\hat{x}_{0}\right)=0$.

We find that

$$
\lim _{\hat{x} \uparrow \bar{x}} R_{\hat{x}}(\hat{x})=f(\bar{x})-\frac{\bar{x}}{T(\bar{x})} f(\bar{x})<0,
$$

since $F(\bar{x})=1$ and $0<\frac{\bar{x}}{T(\bar{x})}<1$. It is immediate that $R_{\hat{x}}(\bar{x})>-\infty$ since $f$ and $T$ are finite. We also find that

$$
\lim _{\hat{x} \downarrow \underline{x}} R_{\hat{x}}(\underline{x})=f(\underline{x})-\frac{\underline{x}}{\lim _{\hat{x} \downarrow \underline{x}} T(\hat{x})} f(\underline{x})+\left(1-\frac{\lim _{\hat{x} \downarrow \underline{x}} T_{\hat{x}}(\hat{x})}{\lim _{\hat{x} \downarrow \underline{x}} T(\hat{x})} \underline{x}\right)\left\{\frac{1}{\lim _{\hat{x} \downarrow \underline{x}} T(\hat{x})}\right\}=\frac{1}{2 \underline{x}},
$$


since

$$
\lim _{\hat{x} \downarrow \underline{x}} T(\hat{x})=\frac{\lim _{\hat{x} \downarrow \underline{x}} \int_{\underline{x}}^{\hat{x}} x d F(x)}{\lim _{\hat{x} \downarrow \underline{x}} F(\hat{x})}=\frac{\lim _{\hat{x} \downarrow \underline{x}} \frac{\partial \int_{\underline{x}}^{\hat{x}} x d F(x)}{\partial \hat{x}}}{\lim _{\hat{x} \downarrow \underline{x}} \frac{\partial F(\hat{x})}{\partial \hat{x}}}=\underline{x},
$$

and

$$
\lim _{\hat{x} \downarrow \underline{x}} T_{\hat{x}}(\hat{x})=\frac{\lim _{\hat{x} \downarrow \underline{x}}\{\hat{x}-T(\hat{x})\} f(\hat{x})}{\lim _{\hat{x} \downarrow \underline{x}} F(\hat{x})}=\frac{\lim _{\hat{x} \downarrow \underline{x}} \frac{\partial\{\hat{x}-T(\hat{x})\} f(\hat{x})}{\partial \hat{x}}}{\lim _{\hat{x} \downarrow \underline{x}} \frac{\partial F(\hat{x})}{\partial \hat{x}}}=1-\lim _{\hat{x} \downarrow \underline{x}} T_{\hat{x}}(\hat{x}),
$$

where L'Hopital's rule applies to each limit. Therefore, $-\infty<\lim _{\hat{x} \uparrow \bar{x}} R_{\hat{x}}(\hat{x})<0<$ $\lim _{\hat{x} \downarrow \underline{x}} R_{\hat{x}}(\hat{x})<\infty$.

Finally, the mean value theorem guarantees the existence of at least one point $\hat{x}_{0} \in$ $(\underline{x}, \bar{x})$ such that $R_{\hat{x}}\left(\hat{x}_{0}\right)=0$, since $R_{\hat{x}}(\hat{x})$ is continuous on $(\underline{x}, \bar{x})$. 